\title{
4H-SiC Microring Resonators for Nonlinear Integrated Photonics
}

\author{
Zheng, Yi; Pu, Minhao; Yi, Ailun; Ou, Haiyan
}

Published in:

Proceedings of 21st European Conference on Integrated Optics

Publication date:

2019

Document Version

Peer reviewed version

Link back to DTU Orbit

Citation (APA):

Zheng, Y., Pu, M., Yi, A., \& Ou, H. (2019). 4H-SiC Microring Resonators for Nonlinear Integrated Photonics. In Proceedings of 21 st European Conference on Integrated Optics

\section{General rights}

Copyright and moral rights for the publications made accessible in the public portal are retained by the authors and/or other copyright owners and it is a condition of accessing publications that users recognise and abide by the legal requirements associated with these rights.

- Users may download and print one copy of any publication from the public portal for the purpose of private study or research.

- You may not further distribute the material or use it for any profit-making activity or commercial gain

- You may freely distribute the URL identifying the publication in the public portal

If you believe that this document breaches copyright please contact us providing details, and we will remove access to the work immediately and investigate your claim. 


\title{
4H-SiC Microring Resonators for Nonlinear Integrated Photonics
}

\author{
Yi Zheng ${ }^{1}$, Minhao $\mathrm{Pu}^{1}$, Ailun $\mathrm{Yi}^{2}$, Xin $\mathrm{Ou}^{2}$, Haiyan $\mathrm{Ou}^{1}$ \\ ${ }^{1}$ DTU Fotonik, Technical University of Denmark, Building 343, DK-2800 Lyngby, Denmark \\ ${ }^{2}$ Institute of Microsystem and Information Technology, Chinese Academy of Sciences, Shanghai, China \\ e-mail: haou@fotonik.dtu.dk
}

\begin{abstract}
We demonstrate efficient four-wave mixing in high $\mathrm{Q} 4 \mathrm{H}-\mathrm{SiC}$ microring resonators. We achieve a four-wave mixing conversion efficiency of $-21.7 \mathrm{~dB}$ in a microring resonator with $79 \mathrm{~mW}$ pump power. Thanks to the strong light confinement in $\mathrm{SiC}$ waveguides with sub-micron cross-section dimensions, a high nonlinear parameter $(\gamma)$ of $12.5 \mathrm{~W}^{-1} \mathrm{~m}^{-1}$ is obtained, from which the nonlinear refractive index $\left(\mathrm{n}_{2}\right)$ of $4 \mathrm{H}-\mathrm{SiC}$ is estimated to be $9.2 \pm 0.4 \times 10^{-19} \mathrm{~m}^{2} / \mathrm{W}$ at telecom wavelengths.
\end{abstract}

Keywords: Nonlinear optics, integrated optics, microresonators.

\section{INTRODUCTION}

An abundance of nonlinear optical effects have been realized in nonlinear integrated optics, which shows great potential for a variety of applications such as high speed signal processing [1], electro-optic modulation, sensing, light amplification, etc [2]. Efficient four-wave mixing (FWM) can also lead to Kerr frequency comb generation in high quality factor $(Q)$ nonlinear microresonators [3]. Therefore, tremendous effort has been made to develop nonlinear integrated material platforms and frequency combs have been achieved in different materials including silicon [4], $\mathrm{Si}_{3} \mathrm{~N}_{4}$ [5], Hydex [6], Diamond [7], AlN [8], AlGaAs [9], etc.

$\mathrm{SiC}$ is also considered to be a suitable candidate for nonlinear integrated optics since it exhibits relatively large Kerr nonlinearity on the order of $10^{-18} \mathrm{~m}^{2} \mathrm{~W}^{-1}[10]$. Besides, its quadratic nonlinearity is also strong $(\sim 30 \mathrm{pm} / \mathrm{V}$ [11]) and multi-photon absorption is absent in $\mathrm{SiC}$ at telecom wavelengths, e.g. $\sim 1550 \mathrm{~nm}$, due to its wide bandgap (2.4 eV-3.2 eV depending on its crystallographic type [12]). Other material properties such as CMOS compatibility, high mechanical stiffness and chemical inertness, and large thermal conductivity also make $\mathrm{SiC}$ an attractive platform. Moreover, point defects in $\mathrm{SiC}$ can be exploited for single-photon sources for quantum applications. In recent years, a few nonlinear processes have been demonstrated in $\mathrm{SiC}$ such as second harmonic generation [13], self-phase modulation [10] and parametric wavelength conversion [14]. In addition, Kerr nonlinearity was also reported in amorphous SiC [15]. Microresonators are key components for enhancing the efficiency of nonlinear processes due to the intra-cavity power enhancement. FWM in 3C-SiC microring resonators has been reported [16], however, the high loss of 3C-SiC microring makes it a relatively inefficient process. For nonlinear processes, high $Q$ microresonators are essential since it offers large enhancement of intracavity optical power. In addition, as the nonlinear parameter $\gamma$ expressed by $2 \pi n_{2} / \lambda \mathrm{A}_{\text {eff }}$ is highly dependent on the effective mode area $A_{\text {eff, }}$ a high- $Q$ microresonator with a small effective mode area is highly desired to achieve efficient nonlinear processes.

We realize efficient wavelength conversion through a FWM process in high- $Q, 4 \mathrm{H}-\mathrm{SiC}$ microring resonators with sub-micron cross-sectional dimension. $-21.7 \mathrm{~dB}$ conversion efficiency is achieved in a $16.5-\mu \mathrm{m}$ microring resonator with $79 \mathrm{~mW}$ pump power. Nonlinear parameter $\gamma$ of $12.5 \mathrm{~W}^{-1} \mathrm{~m}^{-1}$ is obtained in such high-confinement waveguides, which is over three times larger than the value achieved in $3 \mathrm{C}-\mathrm{SiC}$ microresonators. Besides, we also report nonlinear refractive index of $4 \mathrm{H}-\mathrm{SiC}$ at telecom wavelength range as high as $\sim 9.2 \pm 0.4 \times 10^{-19} \mathrm{~m}^{2} / \mathrm{W}$.

\section{FABRICATION AND CHARACTERIZAION}

We applied smart-cut technique to realize SiCOI wafer [17], where $\sim 1.1 \mu \mathrm{m} \mathrm{SiC} \mathrm{layer} \mathrm{was} \mathrm{left} \mathrm{after} \mathrm{the} \mathrm{smart-}$ cut process. A wet oxidation process was applied to thin down the $\mathrm{SiC}$ thickness from $1.1 \mu \mathrm{m}$ to $600 \mathrm{~nm}$ while the roughness was reduced from $\sim 5.27 \mathrm{~nm}$ to $\sim 0.84 \mathrm{~nm}$. The device fabrication on the SiCOI wafer started with pattern definition by electron beam lithography (EBL) in hydrogen silsesquioxane (HSQ) with a multi-pass process [18],[19]. The EBL was performed using a JEOL system (JBX-9500FS) at 100-kV. The HSQ pattern was then transferred to the $\mathrm{SiC}$ layer using a fluorine $\left(\mathrm{SF}_{6}\right)$-based dry etching process in an inductively coupled plasma reactive ion etching (ICP-RIE) machine. After that, an extra wet oxidation was applied to further thin down the $\mathrm{SiC}$ layer thickness to $500 \mathrm{~nm}$ and reduce the roughness for both top and sidewall surfaces. In the end, $\mathrm{SiO}_{2}$ was deposited as the cladding material through Low-Pressure Chemical Vapor Deposition (LPCVD) followed by Plasma-Enhanced Chemical Vapor Deposition (PECVD). 
(a)

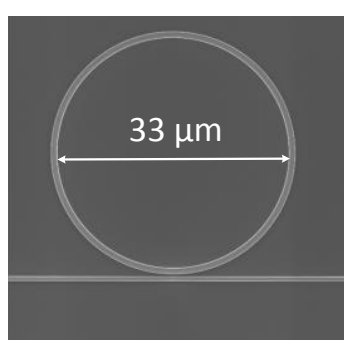

(c)

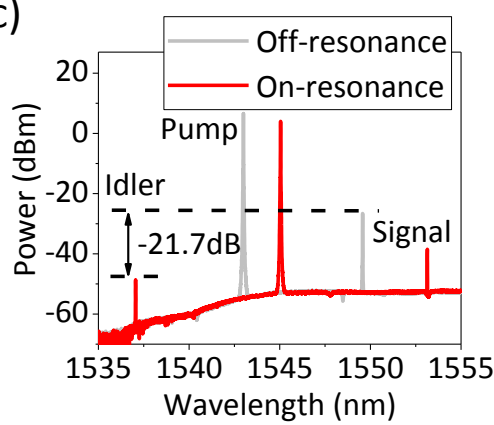

(b)

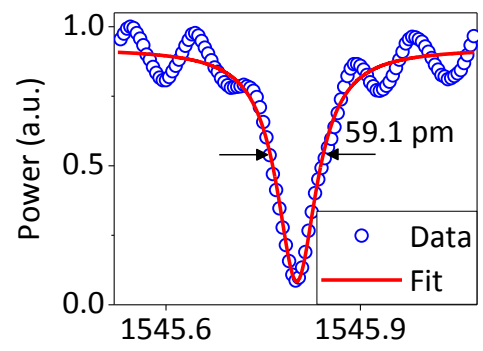

(d)

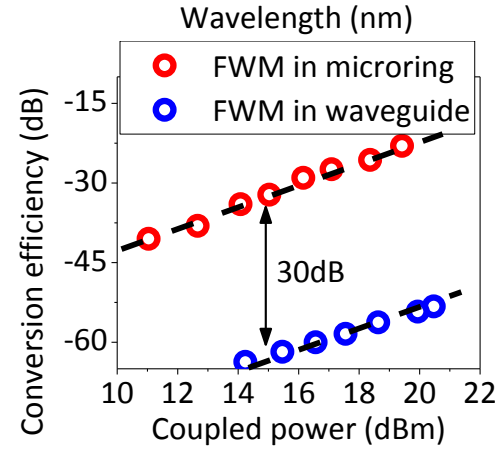

Figure 1. (a) Top-view SEM picture of a fabricated SiC microring resonator with 16.5- $\mu$ m radius. (b) Measured normalized transmission spectrum of an SiCOI microresonator with fitting around $1545 \mathrm{~nm}$. (c) Measured output spectra of the microresonator with the pump and signal waves off-resonance (grey curve) and on-resonance (red curve). (d) Coupled power dependent CE of a resonance in al6.5- $\mu m$ radius SiC microring resonator (red) and a $3 \mathrm{~mm}$ long waveguide (blue).

To perform FWM experiments, we used a $16.5-\mu \mathrm{m}$ radius microring resonator with cross-sectional dimension of $700 \times 500 \mathrm{~nm}^{2}$ (Fig. 1(a)), which is operated in over-coupled condition with a loaded $Q$ of $\sim 26,000$ (the resonance linewidth is shown in Fig. 1(b)). The intrinsic $Q$ of tested resonator was estimated to be $\sim 70,000$. In the FWM experiment, both pump and signal waves were coupled into the resonator. Figure 1(c) shows the output spectra from the resonator when the pump and signal waves are both off-resonance (grey line) and on-resonance (red line). We achieved an overall conversion efficiency (CE), defined by the ratio between the output idler power and input signal power in the bus waveguide of $-21.7 \mathrm{~dB}$ with $79 \mathrm{~mW}$ of pump power. FWM conversion efficiency was also measured in a 3-mm long $\mathrm{SiC}$ waveguide with the same cross-sectional area as the microring resonator. As shown in Fig. 1(d), $30 \mathrm{~dB}$ enhancement of FWM conversion efficiency was achieved. The FWM conversion efficiency of a 104- $\mu \mathrm{m}$-long waveguide (the physical length of tested $16.5-\mu \mathrm{m}$ radius microring resonator) in perfect phasematched condition (in the case of very small signal-pump detuning) can be estimated by $\left(P_{p} \gamma L_{e f f}\right)^{2}$, where $P_{p}$ and $\gamma$ are the estimated intra-cavity power and nonlinear parameter. $L_{\text {eff }}$ is the effective interaction length of the pump and signal in the waveguide defined as $\left(1-e^{-\alpha L}\right) / \alpha$, where $\mathrm{L}$ is the length of the device. The estimated $\mathrm{CE}$ was $-83.5 \mathrm{~dB}$. Therefore, $61.8 \mathrm{~dB} \mathrm{CE}$ gain was obtained at the same pump power level in a microresonator device.
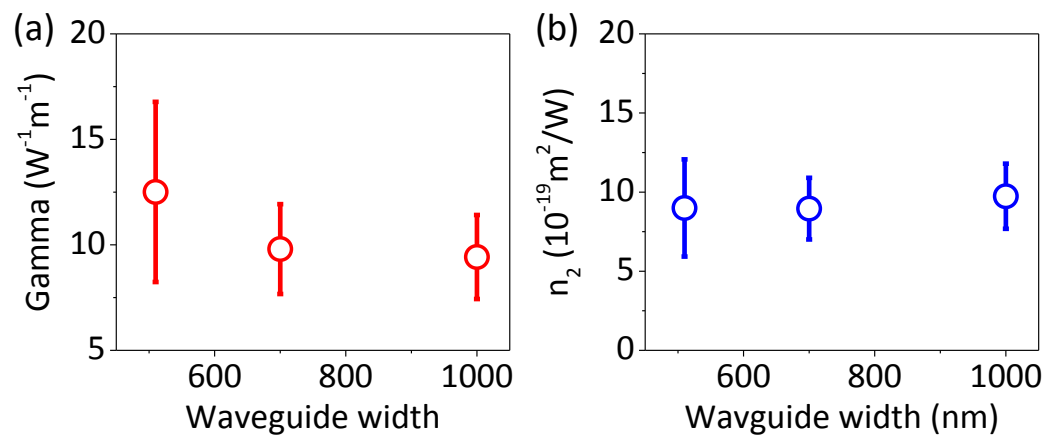

Figure 2.(a) Estimated nonlinear parameter $\gamma$ and (b) nonlinear refractive index $n_{2}$ (blue) based on CE measurement on microring resonators with different cross-sectional areas.

To extract the nonlinear parameter $\gamma$, we measured the CE for 16.5-microring resonators with different waveguide widths (including $510 \mathrm{~nm}, 700 \mathrm{~nm}$ and $1000 \mathrm{~nm}$ ). The red circles in Fig. 2(a) show that the estimated $\gamma$ values are around $12.5,10$ and $9.4 \mathrm{~W}^{-1} \mathrm{~m}^{-1}$ for $500 \mathrm{~nm}$ thick SiC microring resonators with different waveguide widths. By calculating the effective mode areas for these waveguides, we estimated the nonlinear refractive index of $4 \mathrm{H}-\mathrm{SiC}$ at telecom wavelength range is on the order of $9.2 \pm 0.4 \times 10^{-19} \mathrm{~m}^{2} / \mathrm{W}$ (Fig. 2(b)). The obtained $\gamma$ of $12.5 \mathrm{~W}^{-1} \mathrm{~m}^{-1}$ is over three times larger than previous reported result in $3 \mathrm{C}-\mathrm{SiC}$ waveguides [16]. 


\section{CONCLUSIONS}

In conclusion, we demonstrated efficient FWM process in $4 \mathrm{H}-\mathrm{SiC}$ microring resonators. $-21.7 \mathrm{~dB}$ conversion efficiency was achieved in a microring resonator, which is 30-dB higher than a 3-mm long waveguide at the same pump power. Highly nonlinear $\mathrm{SiC}$ waveguides with nonlinear parameter $\gamma$ of $12.5 \mathrm{~W}^{-1} \mathrm{~m}^{-1}$ has been realized, which is higher than previously reported value. The demonstration of this work shows potential for enabling all-optical signal processing functionalities through highly nonlinear $\mathrm{SiC}$ waveguides.

\section{ACKNOWLEDGEMENTS}

The authors acknowledge financial support from the Danish Research Council SPOC (DNRF123) and the National Natural Science Foundation of China (No.: 11705262, 11622545 and U1732268), Frontier Science Key Program of CAS (No.: QYZDY- SSW-JSC032), One Hundred Talent Program of CAS, International Collaboration Project of Shanghai (No.: 16520721100). We thank Assistant professor Jaime Cardenas at University of Rochester for helpful discussion on the smart-cut process in SiC. We also thank DTU Danchip for the support of the fabrication facilities and technologies.

\section{REFERENCES}

[1] M. Pu et al., "Ultra-Efficient and Broadband Nonlinear AlGaAs-on-Insulator Chip for Low-Power Optical Signal Processing," Laser Photon. Rev., vol. 12, no. 12, p. 1800111 , Dec. 2018.

[2] J. Leuthold, C. Koos, and W. Freude, "Nonlinear silicon photonics," Nat. Photonics, vol. 4, no. 8, pp. 535544, Aug. 2010.

[3] P. Del Haye, A. Schliesser, O. Arcizet, T. Wilken, R. Holzwarth, and T. J. Kippenberg, "Optical frequency comb generation from a monolithic microresonator," Nature, vol. 450, no. 7173, pp. 1214-1217, Dec. 2007.

[4] A. G. Griffith et al., "Silicon-chip mid-infrared frequency comb generation,” Nat. Commun., vol. 6, no. 1, p. 6299, Dec. 2015.

[5] J. S. Levy, A. Gondarenko, M. a. Foster, A. C. Turner-Foster, A. L. Gaeta, and M. Lipson, "CMOScompatible multiple-wavelength oscillator for on-chip optical interconnects," Nat. Photonics, vol. 4, no. 1, pp. 37-40, Dec. 2009.

[6] L. Razzari et al., "CMOS-compatible integrated optical hyper-parametric oscillator," Nat. Photonics, vol. 4, no. 1, pp. 41-45, Dec. 2009.

[7] B. J. M. Hausmann, I. Bulu, V. Venkataraman, P. Deotare, and M. Lončar, "Diamond nonlinear photonics," Nat. Photonics, vol. 8, no. 5, pp. 369-374, Apr. 2014.

[8] H. Jung, C. Xiong, K. Y. Fong, X. Zhang, and H. X. Tang, "Optical frequency comb generation from aluminum nitride microring resonator.," Opt. Lett., vol. 38, no. 15, pp. 2810-3, Aug. 2013.

[9] M. Pu, L. Ottaviano, E. Semenova, and K. Yvind, "Efficient frequency comb generation in AlGaAs-oninsulator," Optica, vol. 3, no. 8, p. 823, Aug. 2016.

[10] J. Cardenas et al., "Optical nonlinearities in high-confinement silicon carbide waveguides," Opt. Lett., vol. 40, no. 17, pp. 4138-4141, 2015.

[11] I. J. Wu and G. Y. Guo, "Second-harmonic generation and linear electro-optical coefficients of SiC polytypes and nanotubes," Phys. Rev. B, vol. 78, no. 3, p. 35447, Jul. 2008.

[12] V. E. Chelnokov and A. L. Syrkin, "High temperature electronics using SiC: actual situation and unsolved problems," Mater. Sci. Eng. B, vol. 46, no. 1-3, pp. 248-253, Apr. 1997.

[13] S. Yamada et al., "Second-harmonic generation in a silicon-carbide-based photonic crystal nanocavity.," Opt. Lett., vol. 39, no. 7, pp. 1768-71, Apr. 2014.

[14] J. Cardenas et al., "Parametric Frequency Conversion in Silicon Carbide Waveguides," in CLEO: 2015, 2015, p. SF1D.7.

[15] X. Lu, J. Y. Lee, S. Rogers, and Q. Lin, "Optical Kerr nonlinearity in a high-Q silicon carbide microresonator," Opt. Express, vol. 22, no. 25, pp. 30826-32, Dec. 2014.

[16] F. Martini and A. Politi, "Four wave mixing in 3C SiC ring resonators," Appl. Phys. Lett., vol. 112, no. 25, p. 251110, Jun. 2018.

[17] L. Di Cioccio, Y. Le Tiec, F. Letertre, C. Jaussaud, and M. Bruel, "Silicon carbide on insulator formation using the Smart Cut process,” Electron. Lett., vol. 32, no. 12, p. 1144, 1996.

[18] L. Ottaviano, M. Pu, E. Semenova, and K. Yvind, "Low-loss high-confinement waveguides and microring resonators in AlGaAs-on-insulator," Opt. Lett., vol. 41, no. 17, p. 3996, 2016.

[19] Y. Zheng, M. Pu, H. K. Sahoo, E. Semenova, and K. Yvind, "High-Quality-Factor AlGaAs-On-Sapphire Microring Resonators,” J. Light. Technol., DOI: 10.1109/JLT.2018.2882305, 2018. 